\title{
Perioperative pain treatment for laparoscopic hemicolectomy - \\ An observational prospective study
}

Katrine Tanggaard ', Rune Hasselager², Pia Munk-Madsen², Ismail Gögenur², Thomas F. Bendtsen ${ }^{3}$, Jens Børglum ${ }^{1}$

'Department of Anaesthesiology \& Intensive Care Medicine, Zealand University Hospital, University of Copenhagen, Denmark 2Department of General Surgery, Zealand University Hospital, University of Copenhagen, Denmark

${ }^{3}$ Department of Anaesthesiology \& Intensive Care Medicine, Aarhus University Hospital, Denmark

\section{Background}

Every year 350 patients undergo surgery due to various forms of colorectal cancer at Zealand University Hospital. The vast majority of patients have part of their colon removed, a hemicolectomy. All patients adhere to the 'Enhanced Recovery After Surgery' protocol. For perioperative pain treatment, the patients receive a standardized multimodal analgesic regime. We wanted to evaluate the success of current pain management.

\section{Methods}

After approval from the Danish Data Protection Agency, an observational prospective study of patients undergoing hemicolectomy at Zealand University Hospital from 2016-2017 was conducted. The study investigated if the patients had received the standardized multimodal analgesic regime consisting of IV paracetamol \& IV sufentanil and ropivacaine wound infiltration prior to emergence in the operating theatre. Postoperative pain in recovery was evaluated using numeric rating scale (NRS) 0-10/10 (highest patient reported value in recovery). Total accumulated postoperative opioid consumption at 0-24 hours was registered. All opioids were converted to oral morphine equivalents.

\section{Results}

In total 60 patients were included in the prospective cohort. All patients received the standardized multimodal analgesic regime. Within the first 24 hours post surgery the patients received $51.91 \mathrm{mg}$ \pm 36.22 (mean $\pm S D$ ) of oral morphine equivalents. A total of 97 percent of the patients received opioids within the first 24 hours post surgery either at in recovery or at the wards. Their maximum pain scores in recovery were $3.28 \pm 2.65$ (mean $\pm S D$ ). Table 1 show maximum pain scores and orale morphine equivalents divided by surgical procedure.

\section{Conclusion}

Despite all patients receiving a multimodal analgesic regime and wound infiltration, $97 \%$ of patients received opioids in recovery or at the wards. This calls for a vast improvement in perioperative pain treatment. Therefore we are currently including patients in a randomized doubleblind controlled trial using ultrasound-guided nerve blocks as an addition to the current multimodal analgesic regimen for patients undergoing laparoscopic hemicolectomy.

\section{Surgical procedure}

Lap. sigmoidectomy

Lap. right hemicolectomy

Lap. left hemicolectomy

\section{NRS}

2.9

3.3

3.9

\section{OME/24 hrs (mg)}

45.4

59.9

59.5

Table 1. Averages. NRS = nummercial rating scale $0-10$, highest score reported the post anaesthesia care unit. $\mathrm{OME}=$ oral morphine equivivalents 